\title{
Simulation of thermal shock cracking in ceramics using bond-based peridynamics and FEM
}

\author{
Ilias N. Giannakeas ${ }^{1}$, Theodosios K. Papathanasiou ${ }^{2 *}$, Hamid Bahai ${ }^{3}$ \\ 1,2 Department of Civil and Environmental Engineering, \\ ${ }^{3}$ Department of Mechanical and Aerospace Engineering, \\ Brunel University London, Uxbridge UB8 3PH, UK \\ *Corresponding Author: Email: theodosios.papathanasiou@brunel.ac.uk
}

\begin{abstract}
The effects of moderate intensity 'hot' or 'cold' shock in brittle solids have been extensively studied, while much less is known about thermal shock response during large temperature variations. In this study, a combined finite element - peridynamics numerical procedure is proposed for the simulation of cracking in ceramic materials, undergoing severe thermal shock. Initially, Finite Element nonlinear heat transfer analysis is conducted. The effects of surface convection and radiation heat exchange are also included. Subsequently, the interpolated temperature field is used to formulate a varying temperature induced action for a bond-based peridynamics model. The present model, which is weakly coupled, is found to reproduce accurately previous numerical and experimental results regarding the case of a 'cold' shock. Through several numerical experiments it is established that 'cold' and 'hot' shock conditions give rise to different failure modes and that large temperature variations lead to intensified damage evolution.
\end{abstract}

Keywords: thermal shock cracking; refractory ceramics; bond-based peridynamics; nonlinear heat transfer; fracture

\section{Introduction}

Ceramics and refractories are an extremely diverse family of materials that have met wide applicability across many industries. They exhibit high compressive strength, hardness and melting point, low thermal and electrical conductivity as well as the ability to maintain their properties at elevated temperatures. Due to their excellent performance under elevated temperatures, typical applications include: dies for metal forming, liquid steel technologies, thermal barrier coatings and others [1], [2]. Aluminum oxide $\left(\mathrm{Al}_{2} \mathrm{O}_{3}\right)$ and zirconia $\left(\mathrm{ZrO}_{2}\right)$ are two commonly used engineering oxide ceramics [3]. However, the inherent brittle nature of ceramic materials makes them prone to cracking when subjected to sudden temperature variations. Due to its paramount importance, thermal shock induced cracking in ceramic materials has been studied by many researchers over the last decades. Plentiful studies in the literature, both numerical and experimental, aim to investigate 
the maximum temperature change a brittle material can withstand prior to cracking, thus estimating its thermal shock resistance [4]-[9]. Furthermore, thermal fatigue due to repeated temperature fluctuations is of high academic and industrial interest [10], [11].

Investigations on the thermal induced stress field, have illustrated that specimens undergoing cold shock develop tensile stresses near the boundary and compressive stresses in the interior [7], [8]. The reversed effect is observed during heating of the specimen where tensile stresses develop in the interior. Lu and Fleck [7] presented a systematical classification of solids based on their thermal resistance. Cracking of the material was investigated assuming a pre-existing crack embedded in the area under tension and the resulting stresses were compared with the maximum allowed. Bahr et al. [12] investigated the emerging crack patterns after water quenching of pre-heated quartz and glass plates and simulated the crack evolution using multiple-crack models [8].

Early investigations were carried out assuming pre-existing crack or cracks in the medium. More recent studies on thermal shock employ elaborate lattice [13], nonlocal ([14] and [15]) and gradient ([5] and [16]) models to simulate the initiation and propagation of cracks as well as approaches for the incorporation of microstructural characteristics [17], [18]. Additionally, studies on the induced thermal stresses have illustrated that it is crucial to account for temperature dependent material parameters in the simulations [6], [19]. Usually, materials tend to exhibit a softening behaviour as the temperature increases due to decrease of Young's modulus. As reported in [6], not considering the temperature dependency of the material properties tends to lead to underestimation of the actual thermal shock resistance. In their work, Papathanasiou et al. [19] carried out a detailed investigation on the thermomechanical response of ceramic refractories under extreme temperature changes. The nonlinear heat equation was solved using FEM for a 2D problem, taking into consideration radiation heat exchange and temperature dependant thermal and elastic material properties. The calculated temperature field was subsequently used to determine the induced stress field and the results were compared with those arising from linear models for different values of the Biot number.

In this study, the bond-based peridynamic theory is used for the simulation of fracture in brick-like alumina specimens during a sudden, extreme temperature change. The experimental and numerical results of cold shocked alumina, reported in [15], are compared with the results of the proposed method for validation purposes. Numerical results for cold shock of specimens with temperature dependent properties are derived as well. The model is also used to simulate the fracture of a hot shocked specimen. To the best of the authors' knowledge, numerical simulations for hot shock related analysis are scarcer, while some experimental procedures have been reported to yield results that are not easily reproducible [20].

The present study is organized as follows: Section 2 defines the problem considered and the temperature dependence of alumina's thermal and mechanical properties. In Section 3 the FE scheme employed for the solution of the nonlinear heat transfer problem is presented. The uncoupled peridynamic model and the nondimensional parameters employed are introduced in 
section 4. Section 5 is devoted to the validation of the new solution methodology. This is achieved through comparison with the numerical and experimental results reported in [15]. The effect of including radiative heat transfer and temperature dependent thermal and mechanical material properties is also studied. In section 8 , the hot shock phenomenon is studied and the results are compared with observations from the relevant literature. Concluding remarks and suggestions for future work are included in Section 7.

\section{Geometry and materials}

Thermal shock is divided into two distinct categories: 'hot' shock and 'cold' shock. During a hot shock, the temperature of the material is rapidly increased, while in cold shock the temperature is decreased. Both cases are crucial for the identification of the maximum temperature variations a material can withstand prior to failure. In this study, thin rectangular alumina $\left(\mathrm{Al}_{2} \mathrm{O}_{3}\right)$ specimens, subjected to sudden and extreme temperature variations, will be simulated numerically. For the cold shock case the pre-heated (to a specified temperature $T_{0}$ ) brick is assumed to undergo sudden heat exchange with a surrounding fluid of temperature $T_{\infty}=293.15 \mathrm{~K} \ll T_{0}$. In the hot shock case, the specimen is assumed initially at room temperature $T_{0}=293.15 \mathrm{~K}$ and then heated due to exposure at an ambient with temperature $T_{\infty} \gg T_{0}$. Various values of $T_{0}$ for the cold shock and $T_{\infty}$ for the hot shock will be considered during the simulations.

In all cases heat transfer is assumed to take place only in the $x y$ plane. Figure 1 , illustrates the physical and numerical model under investigation. The specimen's half-length and thickness are $L=5 H$ and $W=0.2 H$ respectively where $H$ is half the height. Taking advantage of the system's symmetry along $y$ and $x$ axes, only one quadrant of the initial domain is simulated. Adiabatic conditions apply to the edges that coincide with lines of symmetry. Convection and radiation loses occur on the other two edges, as shown in Figure 1. Initially the specimen is assumed to be at a uniform temperature $T_{0}$ and stress free. The material is linear, isotropic, homogeneous and in pristine condition at the initial configuration.
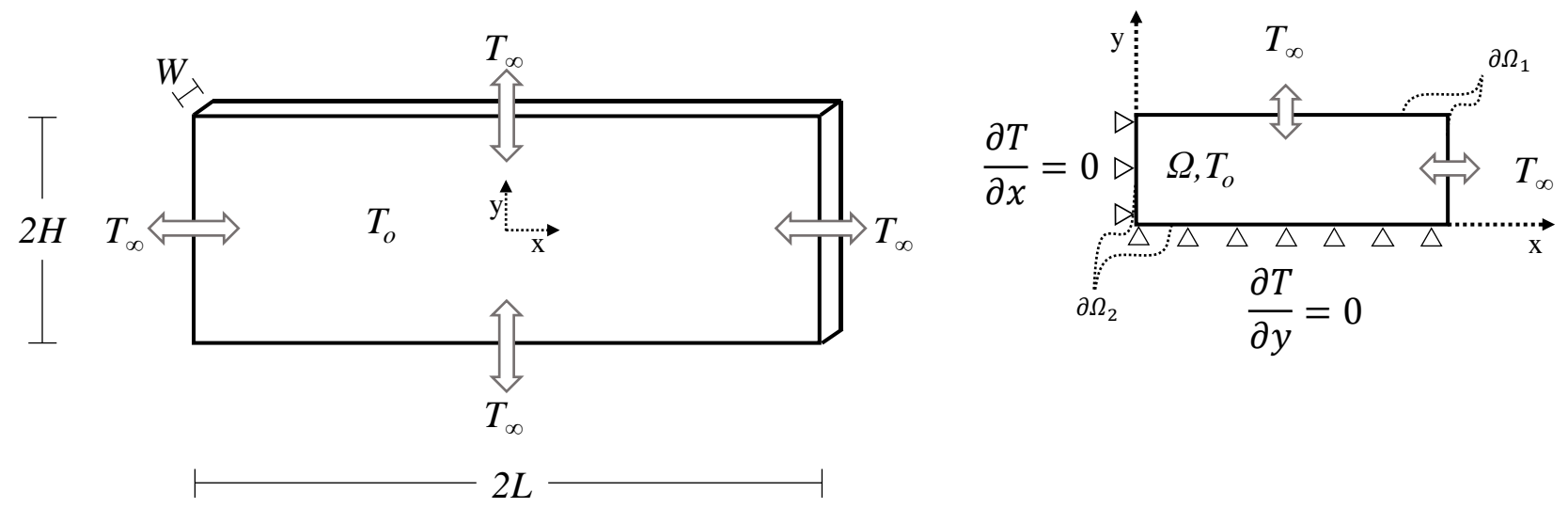

Figure 1: Physical (left) and numerical (right) model of the alumina specimen subjected to 2D heat transfer. 
Due to the severe temperature variations refractories experience throughout their lifetime, it is imperative to include the temperature dependency of the thermal and elastic properties. This need is reflected by the vast scientific effort to accurately capture the variation of these parameters in a wide range of temperatures [21]-[24]. Based on experimental data for polycrystalline alumina, the temperature dependency of the specific heat capacity $c_{p}$ and thermal conductivity $k$ can be approximated with adequate accuracy by inverse power laws [19]:

$$
\begin{gathered}
k(T)=k_{0}+k_{1} T^{-1}, \mathrm{Wm}^{-1} \mathrm{~K}^{-1} \\
c_{p}(T)=c_{0}+c_{1} T^{-1}, \mathrm{Jkg}^{-1} \mathrm{~K}^{-1}
\end{gathered}
$$

where, $T$ is the absolute temperature, $k_{0}, k_{1}, c_{0}$ and $c_{1}$ are constants with values $-4.5536,12227$, 1429.4 and -197620 , respectively. Although more elaborate and accurate functions have been described in [21], equations (1a) and (1b) are adopted here due to their simple form. It is obvious that as temperature increases, the specific heat capacity increases while the thermal conductivity decreases. This is expected, as materials at higher temperatures tend to store heat instead of conducting it [19], [23].

The temperature field is determined considering thermal convection and radiation/irradiation between the material and its environment. Heat exchange can be estimated through the implementation of the Newton cooling law and Stefan-Boltzmann law. To fully characterize heat transfer, two additional parameters must be defined: the emissivity $\varepsilon \in[0,1]$ and the heat transfer coefficient $h$. Heat exchange can then be written as:

$$
q_{\mathrm{surf}}=q_{\mathrm{conv}}+q_{\mathrm{rad}}=h\left(T-T_{\infty}\right)+\varepsilon \sigma\left(T^{4}-T_{s}^{4}\right),
$$

where, $\sigma=5.6710^{-8} \mathrm{~W}^{-2} \mathrm{~K}^{-4}$, is the Stefan-Boltzmann constant and $T_{S}$ is the temperature at the surface of the solid. Following [19], heat loss due to radiation is taken as $\varepsilon=0.80$.

Estimation of the convective heat transfer coefficient $h$, is crucial for the accurate simulation of the heat transfer between alumina and the surrounding medium. Various experimental configurations have been proposed in the literature (e.g. [25], [26]) but as reported in [1], results exhibit high dispersion. Following [15], the value $h=50,000 \mathrm{Wm}^{-2} \mathrm{~K}^{-1}$ is employed, as representative of the heat transfer between a liquid and a solid (water quenching for cold shock or molten steel for hot shock).

The temperature dependence of alumina's mechanical properties needs also to be accounted for. The elastic modulus $E$ and fracture toughness $K_{I C}$, decrease as the temperature increases while, the thermal expansion coefficient $\alpha$ and Poisson's ratio $v$, increase. In the subsequent analyses, the use of the Bond-based peridynamic model restricts the values of the Poisson's ratio to 0.33 for plane stress problems [27]. Nevertheless, the temperature variation of the Poisson's ratio is also included for completeness. Although the Poisson's ratio of the refractory material selected is different than the value enforced by the bond-based peridynamics (which is independent of the temperature 
variation) the results obtained indicate that the prediction of failure due to thermal shock is reasonably accurate. Despite, the temperature dependency of the thermal expansion coefficient being in general nonlinear, a simple linear function can approximate the experimental observations with adequate accuracy [19], [23]. Determining the fracture toughness of a material during the initiation of a crack is difficult [28]. Experimental studies have illustrated that the fracture toughness of high purity alumina tends to decrease as temperature increases. Following the observations reported in [29], the fracture toughness of alumina, expressed in terms of stress intensity, is assumed to decrease linearly as temperature increases. The elastic and mechanical properties of alumina are calculated using:

$$
\begin{gathered}
E(T)=E_{0}\left[1-\lambda_{1}\left(T-T_{0}\right)\right] \\
v(T)=v_{0}\left[1+\lambda_{2}\left(T-T_{0}\right)\right] \\
a(T)=a_{0}\left[1+\lambda_{3}\left(T-T_{0}\right)\right] \\
K_{I C}(T)=K_{0}\left[1-\lambda_{4}\left(T-T_{0}\right)\right],
\end{gathered}
$$

where, $E_{0}=370 \mathrm{GPa}, v_{0}=0.25, a_{0}=6 \cdot 10^{-6} \mathrm{~K}^{-1}$ and $K_{0}=3.3 \mathrm{MPa} \sqrt{\mathrm{m}}$ are the elastic modulus, Poisson's ratio, thermal expansion coefficient and fracture toughness of polycrystalline alumina at room temperature and $\lambda_{1}=1.2 \cdot 10^{-4} \mathrm{~K}^{-1}, \lambda_{2}=6.9 \cdot 10^{-5} \mathrm{~K}^{-1}, \lambda_{3}=9 \cdot 10^{-4} \mathrm{~K}^{-1}$, and $\lambda_{4}=$ $1.567 \cdot 10^{-4} \mathrm{~K}^{-1}$ are constants. Using equations (1a), (1b) and (3a)-(3d), the temperature dependency of the thermal and elastic material properties is illustrated in Figure 2. In the following paragraphs, the fracture process will be related to the critical energy release rate $G_{I C}$. A rough estimate of the fracture toughness (using Linear Elastic Fracture Mechanics for relatively brittle materials like alumina and plane stress assumptions) is $G_{I C}=K_{I C}^{2} / E$.
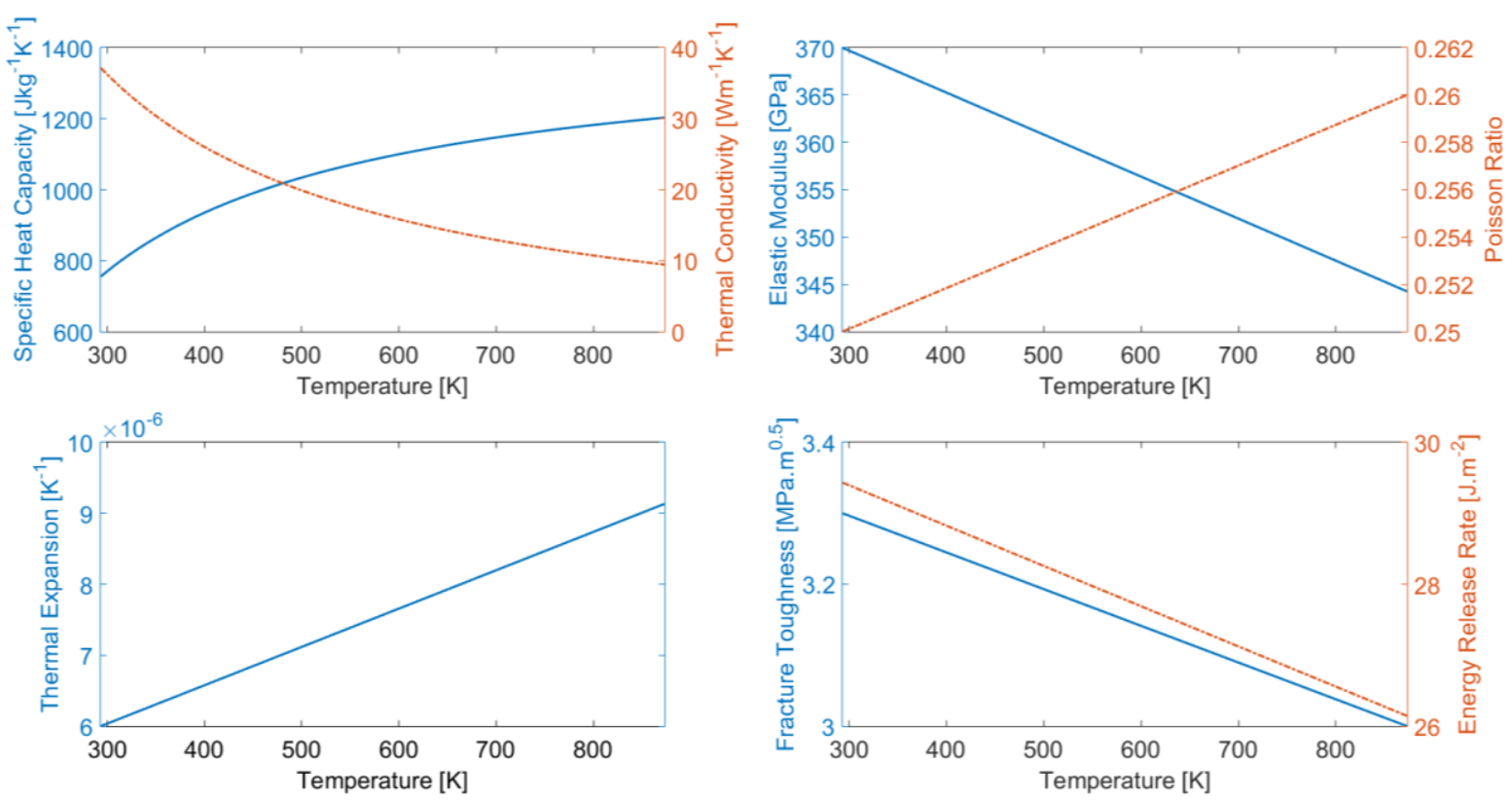

Figure 2: Temperature dependency of thermal and elastic properties for polycrystalline alumina $\left(\mathrm{Al}_{2} \mathrm{O}_{3}\right)$. 


\section{Heat transfer simulation}

When the thermal properties are assumed constant, solution of the mixed boundary problem illustrated in Figure 1, can be obtained directly from available analytical solutions [30]. When the thermal properties are functions of temperature, analytical solutions are not possible and the temperature field needs to be approximated numerically [31]. The Finite Element Method (FEM), is commonly employed for the approximation of complex thermomechanical problems when analytical solutions are not available. Here, Langrage elements with bilinear interpolation of the temperature field are used for the solution of the nonlinear heat transfer problem [19].

Following the derivation presented in [19], the nondimensional spatial and temporal parameters introduced are:

$$
\begin{aligned}
& \hat{\xi}_{x}=H^{-1} x, \\
& \hat{\xi}_{y}=H^{-1} y \\
& \hat{t}=H^{-2} \kappa t
\end{aligned}
$$

where, the accent $\langle\hat{\cdot}\rangle$ is employed to indicate nondimensional values, $\kappa=k_{\text {ref }} /\left(\rho c_{p, \text { ref }}\right)$ is the thermal diffusivity and $k_{\text {ref }}$ and $c_{p \text {,ref }}$ are the thermal conductivity and specific heat capacity at room temperature, respectively. The nondimensional temperature field:

$$
\hat{\theta}=T_{0}^{-1} T
$$

is introduced. The governing equations and FE solution approach adopted are the same as in Papathanasiou et al. [19].

\section{Thermomechanical bond-based peridynamics}

The bond-based peridynamic theory was first introduced by Siling [32] and it is a recently developed non-local theory. Because the formulation of peridynamics is based on a partial integrodifferential equation [33], it is free of displacement derivates and thus suitable for fracture problems where displacement fields are discontinuous. Additionally, there is no need for external phenomenological criteria or a priori knowledge of the final crack path to simulate the propagation and/or the initiation of a crack [34]. The non-local nature of the theory introduces an internal length scale that allows the incorporation of microstructural characteristics [35]. These properties have made peridynamic models very attractive for the simulation of fracture (see e.g. [36], [37], [34], [38]). Here, the applicability of bond-based peridynamics to thermal shock will be evaluated.

Kilic and Madenci [39], [40] were the first to formulate an uncoupled bond-based peridynamic model by introducing a thermal bond stretch in the pairwise force function $\boldsymbol{f}$ and successfully applying their model on fracture problems. Later, Oterkus et al. [41] introduced a fully coupled thermomechanical model for state-based and bond-based peridynamics. The thermal and deformation state of a solid body are interdependent. In certain cases, this interdependency can be 
neglected leading to uncoupled thermomechanical models in which, only the influence of the temperature field on the deformation is present. As a simplification, it is assumed here that the thermal cracking does not affect the heat transfer and the uncoupled solutions will be used. This hypothesis has been used extensively in the literature (eg. [8]), as cracks are expected to appear parallel to the direction of the heat transfer. The temperature field, approximated using the finite element procedure presented in the previous section [19], is used in the peridynamic model. The peridynamic equation of motion can be written as:

$$
\rho \ddot{\boldsymbol{u}}(\boldsymbol{x}, t)=\int_{H_{x}} \boldsymbol{f}\left(\boldsymbol{u}\left(\boldsymbol{x}^{\prime}, t\right)-\boldsymbol{u}(\boldsymbol{x}, t), \boldsymbol{x}^{\prime}-\boldsymbol{x}\right) d V_{x^{\prime}}+\boldsymbol{b}(\boldsymbol{x}, t),
$$

where, $\boldsymbol{x}$ is the position of a material particle, $H_{x}$ is the neighbourhood of $\boldsymbol{x}$ within which it interacts with other particles, $\boldsymbol{x}^{\prime}$ is another particle in $H_{x}, \boldsymbol{f}$ is the pairwise force function and $\boldsymbol{b}$ is the external body force field. In practice $H_{x}$ is assumed to be a generalized ball of radius $\delta$ (peridynamic horizon).

Following [42], the pairwise force function of an isotropic material under temperature change, can be written as:

$$
\boldsymbol{f}(\boldsymbol{\eta}, \xi)=(c s-c a \theta) \frac{\boldsymbol{\eta}+\xi}{|\boldsymbol{\eta}+\xi|}=\left(c \frac{|\boldsymbol{\eta}+\xi|-|\xi|}{|\xi|}-c a \theta\right) \frac{\boldsymbol{\eta}+\xi}{|\boldsymbol{\eta}+\xi|},
$$

where, $s$ is the bond stretch, $\boldsymbol{\xi}=\boldsymbol{x}^{\prime}-\boldsymbol{x}$ is the relative position vector, $\boldsymbol{\eta}=\boldsymbol{u}^{\prime}-\boldsymbol{u}$ is the relative displacement vector, $a$ is the thermal expansion coefficient, $\theta$ is the temperature change and $c=9 E /\left(\pi \delta^{3} W\right)$ is the bond constant for plane stress. The value of the bond constant is derived by equating the peridynamic strain energy density with its counterpart from classical elasticity [28]. Since $c$ is assumed to be constant, peridynamic bonds describe a linear force-stretch $(\boldsymbol{f}-s)$ relationship, analogous to an elastic spring.

Breaking of the peridynamic bonds takes place when the bond stretch $s$ exceeds a maximum allowable stretch, denoted $s_{0}$. This limit can be related to the critical energy release rate $G_{I C}$, and for a plane stress problem is expressed as [38]:

$$
s_{0}=\sqrt{\frac{4 \pi G_{I C}}{9 E \delta W}}
$$

A bond is considered broken if the criterion $s\left(t^{\prime}, \xi\right)-\alpha \theta \leq s_{0} \forall 0<t^{\prime}<t$ is violated and it is removed from subsequent calculations, changing the stiffness matrix of the structure [39]. Broken bonds are tracked using a Boolean scalar-valued function, $\mu$ [39].

Damage in the solid is quantified through the usage of a local damage index $\phi$ defined as: 
Published in Journal of the Journal of the European Ceramic Society, Volume 38, Issue 8,

$$
\phi(\boldsymbol{x}, t)=1-\frac{\int_{H} \mu(t, \boldsymbol{\eta}, \boldsymbol{\xi}) d V^{\prime}}{\int_{H} d V^{\prime}}
$$

with $0 \leq \phi(x, t) \leq 1$. The local damage is essentially an index that describes the remaining unbroken bonds of a particle compared to the initial number of bonds.

Following the collocation method described in [35], the initial domain of the problem is discretized into subdomains and, combining equations (6) and (7), the uncoupled peridynamic equation of motion is approximated numerically through a finite summation as:

$$
\rho \ddot{\boldsymbol{u}}\left(\boldsymbol{x}_{i}, t\right)=\sum_{j=1}^{M}(c s-c a \theta) \frac{\boldsymbol{\eta}_{(i)(j)}+\boldsymbol{\xi}_{(i)(j)}}{\left|\boldsymbol{\eta}_{(i)(j)}+\xi_{(i)(j)}\right|} V_{j}+\boldsymbol{b}_{\boldsymbol{i}}\left(\boldsymbol{x}_{\boldsymbol{i}}, t\right)
$$

where, $\boldsymbol{x}_{\boldsymbol{i}}$ is the $i^{\text {th }}$ collocation point in the original domain, $M$ is the total number of subdomains that are included within the horizon of the $i^{\text {th }}$ point, $V_{j}$ is the volume of the $j^{\text {th }}$ subdomain and $\boldsymbol{\eta}_{(i)(j)}$ and $\xi_{(i)(j)}$ are the relative displacement and position of the $i^{t h}$ and $j^{\text {th }}$ collocation points respectively. A more advanced discretization method has been proposed by Kilic and Madenci in [42] where the aforementioned discretization can be extracted as a special case.

Boundary conditions are enforced by adding a fictitious material layer at the boundary. In [43], Macek and Silling suggest that the thickness of such layer should be equal to $\delta$. Furthermore, volume correction and surface correction strategies are adopted to account for nodes that are only partially within the horizon and to alleviate the "skin effect" [35], respectively. The methodologies are described in [44] and [35].

In the following paragraphs, both the transient and the static solution of equation (10) are used. Various approaches have been suggested in the literature for the time integration of equation (10). Here the explicit forward and backward difference technique suggested in [37] is employed. At each node $i$, the velocity is updated using the acceleration and the velocity of the node at the current step through the forward Euler method:

$$
\dot{\boldsymbol{u}}_{i}^{n+1}=\ddot{\boldsymbol{u}}_{i}^{n} \Delta t+\dot{\boldsymbol{u}}_{i}^{n}
$$

where, $n$ and $n+1$ denote the current and next time step respectively, $\dot{\boldsymbol{u}}$ is the velocity, $\ddot{\boldsymbol{u}}$ is the acceleration and $\Delta t$ is the time step. The nodal displacements can then be computed using the backward Euler method:

$$
\boldsymbol{u}_{i}^{n+1}=\dot{\boldsymbol{u}}_{i}^{n+1} \Delta t+\boldsymbol{u}_{i}^{n}
$$

Although explicit methods are straightforward to use, they are only conditionally stable. Using the definition of linearized bond-based peridynamics [45], it was shown in [38] that for the algorithm to be stable, the maximum time step must be limited by: 
Published in Journal of the Journal of the European Ceramic Society, Volume 38, Issue 8,

$$
\Delta t \leq \sqrt{\frac{2 \rho}{\sum_{j=1}^{N} \frac{c}{\left|\boldsymbol{x}_{j}-\boldsymbol{x}_{i}\right|} V_{j}}}
$$

In many problems, the solution refers to equilibrium states and static solutions are required. Neglecting inertia forces and setting:

$$
g(\boldsymbol{u})=\sum_{j=1}^{M}(c s-c a \theta) \frac{\boldsymbol{\eta}_{(i)(j)}+\xi_{(i)(j)}}{\left|\boldsymbol{\eta}_{(i)(j)}+\xi_{(i)(j)}\right|} V_{j}+\boldsymbol{b}_{i}\left(\boldsymbol{x}_{\boldsymbol{i}}, t\right) .
$$

Although each bond is assumed to behave linearly, the final system of equations (14) is nonlinear. Zaccariotto et al. [27] suggested the implementation of a full Newton-Raphson method for the approximation of $g(\boldsymbol{u})=0$.

Using the characteristic length and time scales presented in the previous section, the position vector $\widehat{\boldsymbol{x}}$ and the displacement vector $\widehat{\boldsymbol{u}}$, are defined as $\widehat{\boldsymbol{x}}=H^{-1} \boldsymbol{x}$ and $\widehat{\boldsymbol{u}}=H^{-1} \boldsymbol{u}$. The following nondimensional values can then be defined:

$$
\begin{gathered}
\widehat{\hat{\boldsymbol{u}}}=\left(\frac{k_{\mathrm{ref}}}{c_{p, \mathrm{ref} \rho} \rho}\right)^{-2} H^{3} \ddot{\boldsymbol{u}}, \\
\hat{c}=\frac{9 E}{\pi \hat{\delta}^{3} \widehat{W}}=\frac{9 E}{\pi \delta^{3} W} H^{4}=c H^{4}, \\
\widehat{V}=\widehat{\Delta x}^{2} \widehat{W}=H^{-3} V,
\end{gathered}
$$

where, $\widehat{\Delta x}$ is the lattice spacing considered for the spatial discretization. Using Eqs. (15a-15c) in Eq. (10), the dimensionless uncoupled equation of motion is obtained as:

$$
\frac{k_{0}{ }^{2}}{\rho c_{0}{ }^{2} H^{2}} \widehat{\ddot{\boldsymbol{u}}}\left(\boldsymbol{x}_{i}, t\right)=\sum_{j=1}^{M}(\hat{c} s-\hat{c} a \hat{\theta}) \frac{\widehat{\boldsymbol{\eta}}_{(i)(j)}+\widehat{\boldsymbol{\xi}}_{(\boldsymbol{i})(j)}}{\left|\widehat{\boldsymbol{\eta}}_{(\boldsymbol{i})(j)}+\widehat{\boldsymbol{\xi}}_{(i)(j)}\right|} \widehat{V}_{j},
$$

where the external body forces $b_{i}$ have been excluded as they are not relevant to the problems considered.

\section{Cold shock and verification of the model}

The sudden nature of material cracking during cold shock has impaired scientists to experimentally observe and monitor the phenomenon [25]. Additionally, the rapid fracture of the material creates complex crack patterns that are difficult to simulate when implementing traditional continuum fracture mechanics. The tensile stresses developing on the surface of the material leads to the initiation of surface breaking cracks [7]. Many methodologies have been proposed for the approximation of the stress field and stress intensity factors. More recent studies, also incorporate microstructural characteristics as well as effects due to the presence of micro cracks in the medium 
[18], [46]. A distinctive characteristic of the final crack patterns that emerge, is the varying crack length that forms hierarchical classes of cracks [5], [15].

The efficiency of bond-based peridynamics for numerical simulations of thermal shock cracking is evaluated by comparing the present methodology with the numerical and experimental results for $\mathrm{Al}_{2} \mathrm{O}_{3}$ specimens reported in [15]. To ensure compatibility and comparability of the results, the temperature dependency of the thermal and mechanical properties is initially neglected and the material parameters used in [15] are employed. Additionally, radiative heat transfer is also neglected. Table 1 , summarizes the parameters used.

Table 1: Temperature independent thermal and mechanical properties employed as specified in [15].

\begin{tabular}{ccc}
\hline \multicolumn{3}{l}{ Thermal and Mechanical Properties } \\
\hline$E$ & 370,000 & $\mathrm{Mpa}$ \\
$\rho$ & 3980 & $\mathrm{~kg} / \mathrm{m}^{3}$ \\
$G$ & 24.3 & $\mathrm{~J} / \mathrm{m}^{2}$ \\
$h$ & 50,000 & $\mathrm{~W} /\left(\mathrm{m}^{2} \cdot \mathrm{K}\right)$ \\
$k$ & 31 & $\mathrm{~W} /(\mathrm{m} \cdot \mathrm{K})$ \\
$c$ & 880 & $\mathrm{~J} /(\mathrm{Kg} \cdot \mathrm{K})$ \\
$\alpha$ & $7.510^{-6}$ & $\mathrm{~K}^{-1}$ \\
\hline
\end{tabular}

Due to the geometry of the model, tensile stresses at the surface of the specimen are virtually uniform along the length. For that reason, to avoid de-bonding of wide areas of the upper surface and accurately capture the crack nucleation sites, sufficiently small time-steps must be used, otherwise cracks become time-step dependent. The transient solution allows for the extraction of dynamic characteristics. However, due to the restrictive maximum time step calculated from equation (13), dynamic simulation of the whole phenomenon is computationally unfeasible. Hence, the transient solution of the peridynamic equation of motion is used to simulate crack nucleation and subsequently an incremental static solution (ignoring inertia effects) is used for the propagation phase till the final arrest. Comparisons with similar numerical and experimental results from the relevant literature, presented in the following, indicate that this approach leads to results of reasonable accuracy. To accelerate the simulation the loading (temperature) increment was increased during the static analysis, following a geometric sequence of $n$ steps with common ratio, $r$. That is the discrete form of Eq. (14) is solved using the temperature distribution, calculated from the FEM solution, at time instants $\Delta \hat{t}_{\mathrm{n}}=\Delta \hat{t}_{0} r^{n-1}$. The initial time step during the transient solution is $\Delta \hat{t}_{0}=3.010^{-9}$ and the maximum time step $\Delta \hat{t}_{\max }=0.05$. The nondimensional time step parameters $\Delta \hat{t}_{\mathrm{n}}, \Delta \hat{t}_{0}$ and $\Delta \hat{t}_{\max }$ are also defined using equation (4c). 


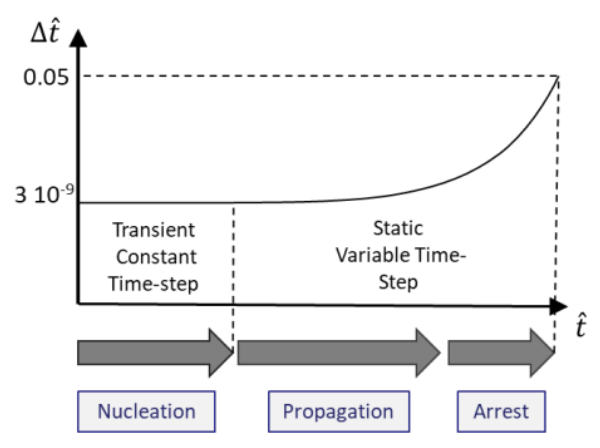

Figure 3: Variation of the time-step during the simulation of cold shock.

Figure 3 illustrates the solution implemented during each phase, as well as the corresponding timestep. The geometry of the specimen is $50 \mathrm{~mm} \times 10 \mathrm{~mm} \times 1 \mathrm{~mm}$ and only one quarter of the domain is considered. The half-height of the specimen is $H=5 \mathrm{~mm}$. The solution domain is discretized assuming 52,416 particles corresponding to a grid spacing $\widehat{\Delta x}=0.01$. A fictitious material layer of length $\hat{\delta}$ is added to enforce the boundary conditions and the peridynamic horizon is set to $\hat{\delta}=$ $3.015 \widehat{\Delta x}$, following observations on macroscale problems reported in the literature [38], [47], [48]. The simulation is carried out for a total time $\hat{t}_{\text {total }}=1$. Setting the appropriate parameters to 0 in Papathanasiou et al. [19], the linear heat transfer problem is retrieved. A 200x40 structured grid was implemented for the FEM discretization. The temperature at each peridynamic particle was then determined using linear interpolation (Figure 4) on the results from the FEM.

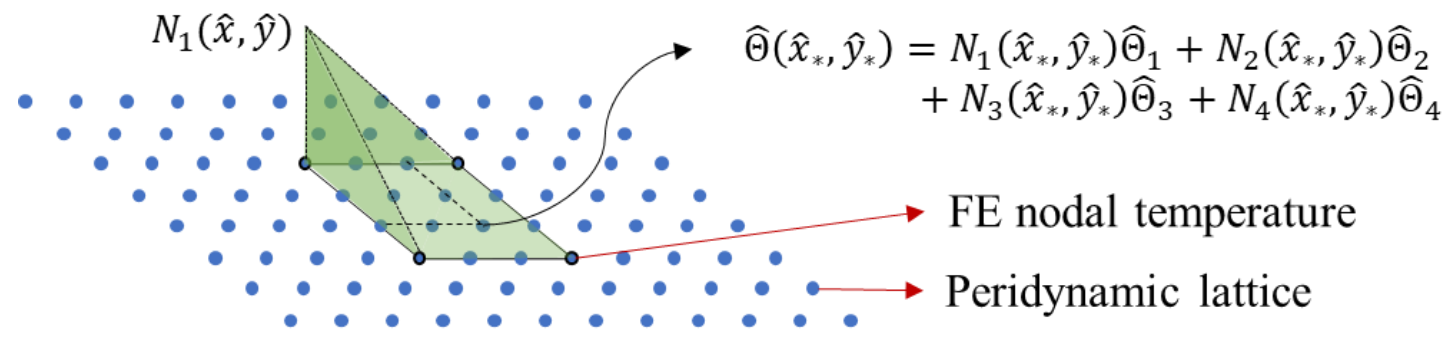

Figure 4: Qualitative illustration of the temperature field interpolation at the peridynamic particles.

First, the evolution of the thermal shock induced cracks in an alumina specimen preheated at $773.15 \mathrm{~K}$ and quenched into a fluid at room temperature $\left(T_{\infty}=293.15 \mathrm{~K}\right)$, is investigated. In Figure 5 , the initiation, propagation and crack patterns are illustrated at various time steps. In the left column, the displacement of each peridynamic particle in the lattice is displayed (displacements have been magnified by a factor of 100). In the right column, the local damage index $\varphi$ is illustrated to enhance visualization of the crack positions. 

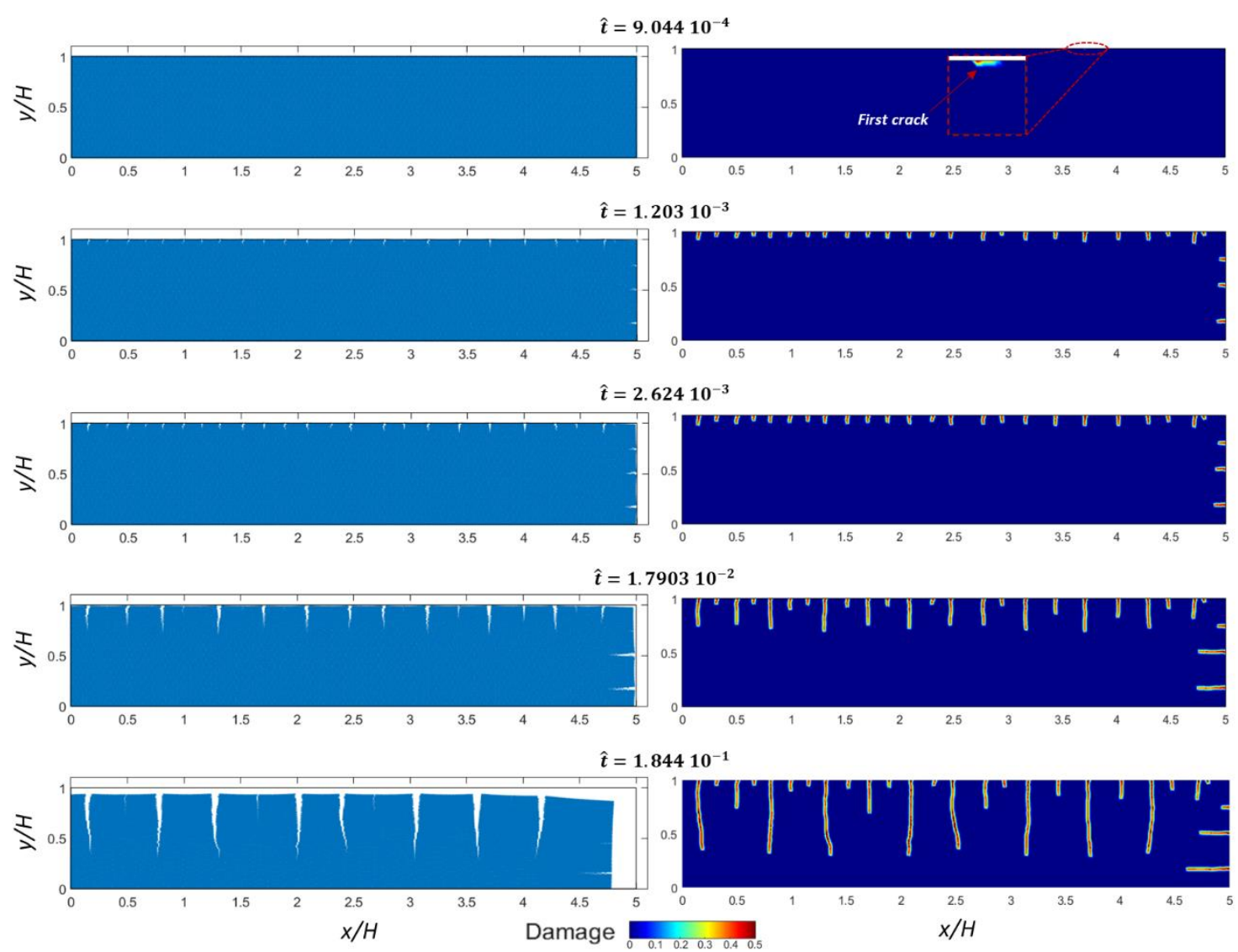

Figure 5: Crack evolution for a specimen preheated at 773.15K and quenched into water at ambient temperature (293.15K).

According to the current simulations, first crack nucleation takes place at approximately $\hat{t}=$ $9.04410^{-4}$ while the last one nucleates at $\hat{t}=1.20310^{-3}$ (first two rows of Figure 5). These time instants indicate the initiation and the completion of the nucleation phase. It is noted that after a crack nucleates, it does not start to propagate towards the interior of the material till after all cracks have emerged. This happens after $\hat{t}=1.20310^{-3}$ and at $\hat{t}=2.62410^{-3}$ the propagation of the cracks is illustrated. Very small variations in the crack lengths at the initial stages result to the formation of a periodic and hierarchical crack pattern, that is distinctive of cold shocked specimens. This is illustrated at $\hat{t}=1.790310^{-2}$, where the hierarchical classes are evident. The mechanism responsible for this pattern is stress flow deviation from the short cracks due to shielding by the larger ones. At $\hat{t}=1.84410^{-1}$, the peridynamic model predicts that crack propagation has been terminated for all cracks and three crack length classes can be identified in the final pattern.

Two additional cases were examined using the peridynamic model, setting the initial temperature $T_{0}$ at $673.15 \mathrm{~K}$ and $873.15 \mathrm{~K}$. When such large temperature differences are considered, nonlinear effects related to thermal dependent moduli and radiation heat transfer need to be included. For that reason, all the above examples have been simulated using the nonlinear heat transfer model as well. The constant properties presented in Table 1 are now replaced by those illustrated in Figure 2. Furthermore, due to the nonlinearities in the heat transfer equations, the finite element mesh was 
increased to 50,000 elements to accurately approximate the temperature variation. The solution procedure is otherwise identical to the one followed in the previous section. The strong influence of temperature depended material properties on the thermal stresses has been documented in the literature [6], [19].
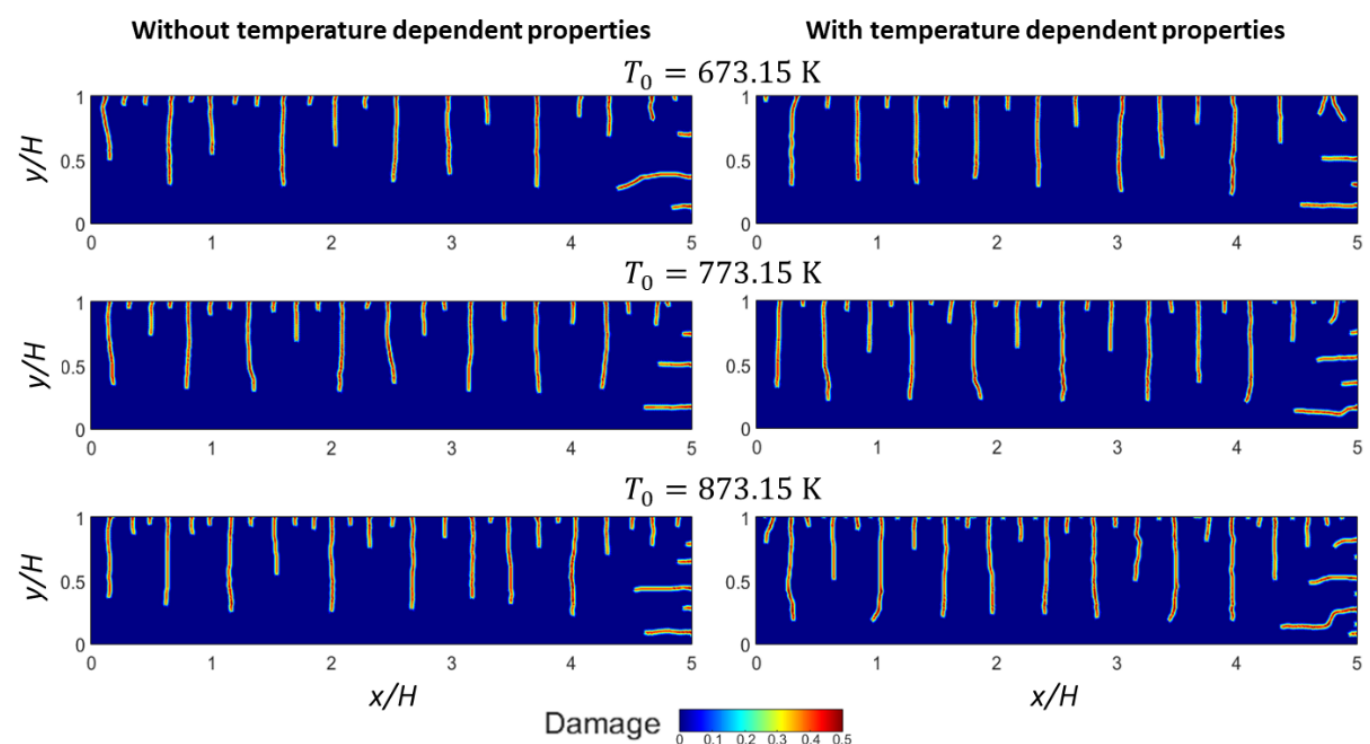

Figure 6: Comparison of the final crack patterns after the introduction of the temperature dependent material properties $(\hat{t}=1.00)$.

Figure 6 offers a comparison between the results from the peridynamic model, with (right column) and without the temperature dependency (left column). Each row in Figure 6 depicts the local damage index at $\hat{t}=1.00$ corresponding to the initial temperature $T_{0} 673.15 \mathrm{~K}, 773.15 \mathrm{~K}$ and 873.15 K respectively. It is evident that for higher initial temperatures, the heat flux within the material intensifies and the crack spacing is decreased, leading to an increase in the total number of cracks. This agrees well with the experimental and numerical results presented in [15] as well as similar observations in the literature (e.g. [4], [10], [14]). The transient algorithm successfully predicted that cracks will nucleate on the material boundary. Subsequently, the static algorithm predicted multi-crack propagation till their final arrest. The spacing of the cracks tends to be even across the boundary and final crack patterns illustrate hierarchical and periodic classes. Increasing the initial temperature $T_{0}$, the density of the crack patterns and the length of the longest crack class increases [10]. In the cases presented here, it was possible to clearly identify three hierarchical classes. When the temperature dependency of the thermal and mechanical material properties is included into the simulations, the length of the longest crack level is increased, while the lower crack levels still follow the distribution observed experimentally [15].

When the temperature dependency is not included, the time instant of first nucleation is $\hat{t}=$ $16.5210^{-4}, \hat{t}=9.0410^{-4}$ and $\hat{t}=5.7610^{-4}$ for initial temperature $T_{0}=673.15 \mathrm{~K}, T_{0}=$ $773.15 \mathrm{~K}$ and $T_{0}=873.15 \mathrm{~K}$, respectively. In the cases considered, the peridynamic model predicts that cracks initiate when the temperature difference on the surface of the material is 
approximately $106{ }^{\circ} \mathrm{C}$. When the temperature dependency is included, the first crack appeared at $\hat{t}=17.0610^{-4}, \hat{t}=6.2510^{-4}$ and $\hat{t}=3.1610^{-4}$ for initial temperature $T_{0}=673.15 \mathrm{~K}$, $T_{0}=773.15 \mathrm{~K}$ and $T_{0}=873.15 \mathrm{~K}$, respectively. After the introduction of the variable material properties, steeper temperature gradients develop in the interior of the material and thus, thermal stresses increase. This explains why cracks appeared sooner compared to the constant properties. At $T_{0}=673.15 \mathrm{~K}$, the first crack appears at comparable time in both cases due to the higher value of the fracture toughness assumed here.

Qualitatively, the bond-based peridynamic model presented here produces crack patterns that are in close agreement with the numerical and experimental results presented in [15]. Differences between the models can be traced back in the use of a transient solution for the first part of the simulation and the limitation on the Poisson ratio (here $v=0.33$ ). Finally, as mentioned earlier, both the present linear temperature peridynamics model and the numerical procedure (based again on linear heat transfer) presented in [14] underestimate the maximum length of the longest cracks. This shortcoming is remedied by the incorporation of nonlinear effects in the heat transfer model.

Aiming for a more detailed comparison, using the results presented in [15], the dimensionless crack length, defined as $\hat{l}=l / H$, is computed for all the initial temperatures considered. Following [14] the comparison presented in Figure 7 , is carried out using the ratio $N / N_{\text {tot }}$, where, $N\left(\hat{l}_{1}, \hat{l}_{2}\right)$ is the number of cracks with crack length $\hat{l}_{1} \leq \hat{l} \leq \hat{l}_{2}$ and $N_{\text {tot }}$ is the total number of cracks. It is thus possible to compare the length of the cracks for each temperature and the number of cracks in each length level and identify the crack clusters that define the hierarchical levels. 

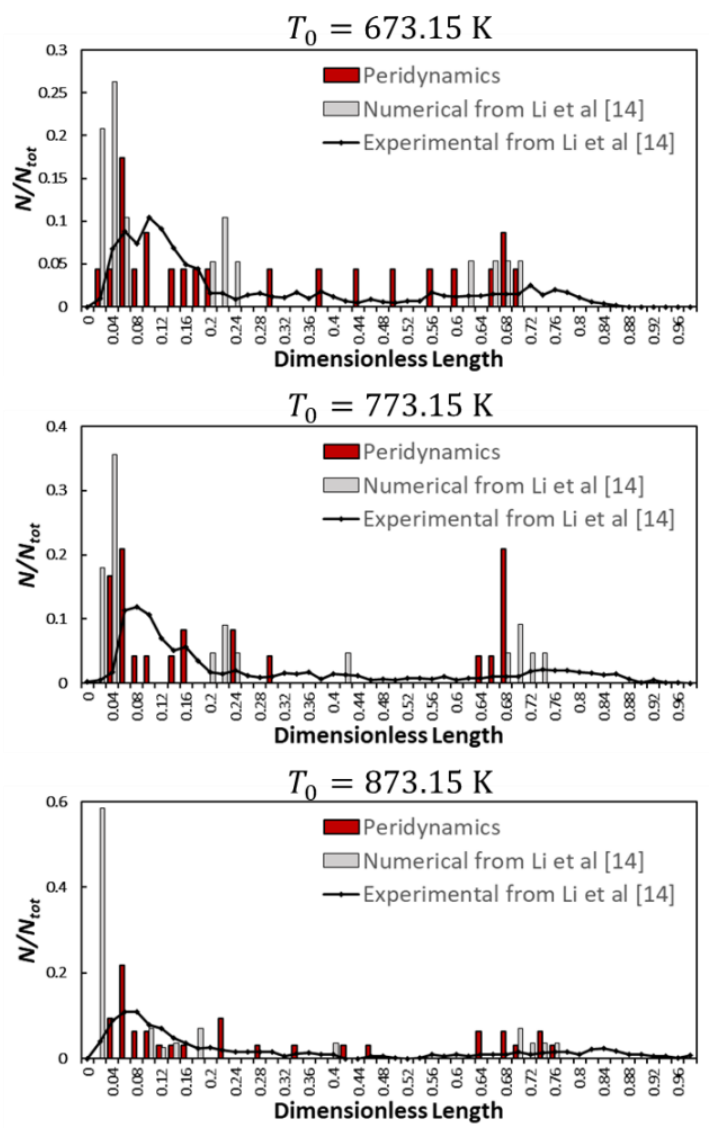

Figure 7: Histograms comparing the number of cracks at each length level between the numerical and experimental results presented in [15] and here.

Commenting on Figure 7, it is identified that the crack lengths in each hierarchical level, as derived from the numerical procedure suggested in [15], exhibit very small dispersion with respect to a central value. At the same time, these length class clusters appear to be slightly misplaced compared to the experimental results found in [14], (gray bars and black line in Figure 7). On the other hand, the crack lengths produced with the peridynamic model exhibit a wider distribution that in the mean sense appears to describe better the experimental observations. In the case of the short hierarchical level specifically, the central value around which the numerical results of [15] cluster, underestimate the experimental findings. However, the results of the proposed method fit better underneath the curve.

As the initial temperature increases, the length of the shortest hierarchical level shifts to the left. This effect was captured by both numerical models, with the peridynamic model leading to better approximations. Although both models predict that the crack length of the longest hierarchical level increases with increasing initial temperature, based on the available experimental results, both models underestimate the final nondimensional lengths of the longest cracks. In this case, the method presented in [15] produces slightly better approximations. This shortcoming is improved after the introduction of the nonlinear effects related to thermal dependent moduli and radiation heat transfer, as illustrated in Figure 8. 

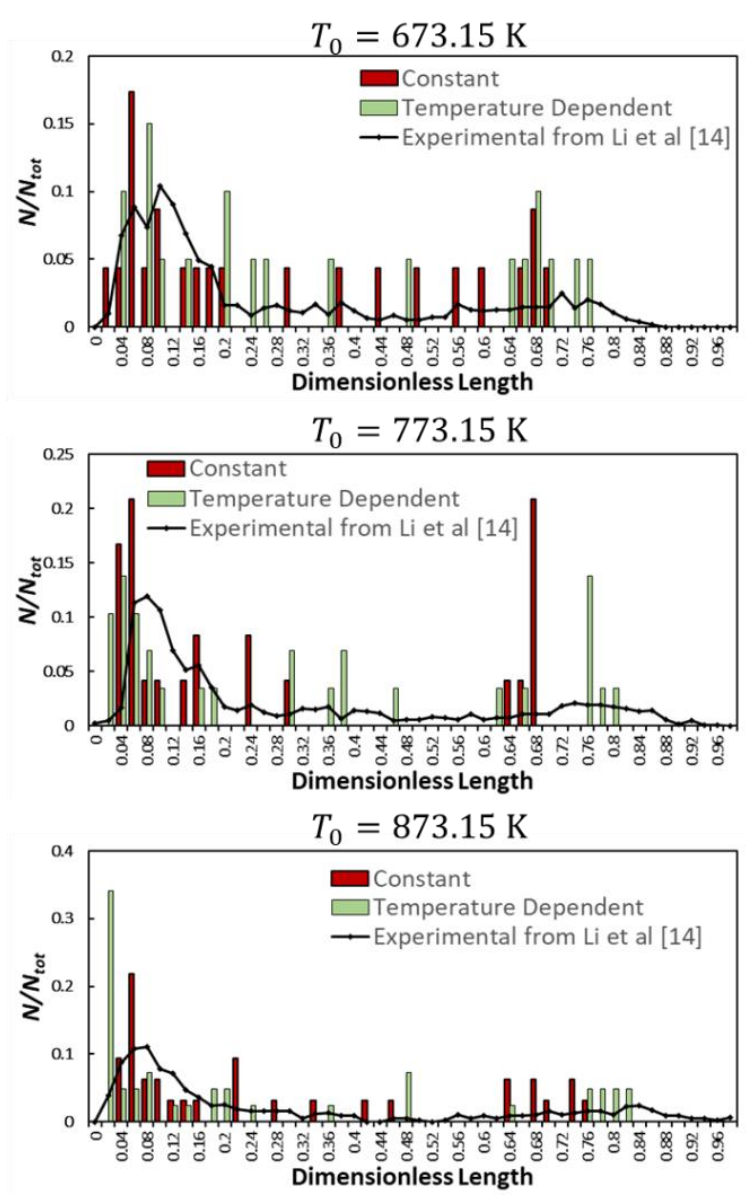

Figure 8: Comparison of the number of cracks at each length level before and after the introduction of the temperature dependent material properties.

In Figure 8, the experimental data presented in [14] (black line) are plotted against the ones derived by the present FEM-peridynamics method. Apart from the linear heat transfer model (red bars), the more realistic nonlinear heat transfer based results are shown using green bars. It is evident from the three plots in Figure 8 that the fully nonlinear model can predict better the formation of larger cracks. The clusters of green bars in higher values of the nondimensional length appear to follow more accurately the line produced by experimental data. The methodology proposed here can approximate the experimental findings over the full spectrum of crack lengths for various initial temperatures.

Concluding the analysis of cold shock, it is necessary to mention that the rapid evolution of cracks is one of the key fracture characteristics of cold shocked specimens. The use of the transient solution of the peridynamic equation of motion during the nucleation phase, allows the evaluation of crack propagation velocities. In the following, the average crack propagation velocity is estimated for the first crack that nucleates, at each initial temperature $T_{0}$, when the temperature dependent properties are used. 
For $T_{0}=673.15 \mathrm{~K}$, the first crack nucleates at $\hat{t}_{1}=1.705910^{-3}$ and propagates till $\hat{t}_{2}=$ $1.706110^{-3}$, for a total length of $\hat{l}=0.105$. The average velocity, expressed as a dimensional value for convenience, is approximately $v_{\text {average }}=1113 \mathrm{~m} / \mathrm{s}$. Similarly, for $T_{0}=773.15 \mathrm{~K}$ and $T_{0}=873.15 \mathrm{~K}$, the average velocities are $v_{\text {average }}=1033 \mathrm{~m} / \mathrm{s}$ and $v_{\text {average }}=719 \mathrm{~m} / \mathrm{s}$, respectively. Typical Rayleigh wave speeds $c_{R}$, for alumina materials are in the region of $5858 \mathrm{~m} / \mathrm{s}$ [49]. The results obtained from the numerical simulations suggest propagation velocities well below $c_{R}$. In [50], Rosakis notes that although the Rayleigh wave speed is the maximum, theoretically attainable, crack velocity for Mode I fracture of ceramics, experimental observations suggest only lower velocity levels are actually attainable. When the crack tip velocity is within the range of approximately $0.35 \sim 0.5 c_{R}$, crack branching is observed. The range of the crack velocities predicted from the peridynamic model during cold shock are below the crack branching velocities and agree with the straight crack patterns observed during experiments. Therefore, the results presented appear to be in agreement with the expected values and provide insights on the rapid nature of the phenomenon.

\section{Hot shock simulation}

In applications like metal forming and gas turbines, refractories are subjected to extremely high temperatures. During a hot shock, the surface of the material tries to expand and compressive stresses develop near its boundaries while tensile stresses develop in the interior of the material [7], [19]. Due to the tensile stresses that develop in the interior, cracks initiate inside the material and propagate towards its boundary [6].

Various techniques have been suggested in the literature to reproduce damage in specimens due to hot shock, under laboratory conditions. These set ups however are more demanding compared to the ones used for cold shock. Open flame burners are probably the simplest set up but the supplied heat flux is not easy to be quantified accurately [4], [51]. It is noted (as also mentioned in [20]) that the ASTM standardized procedure for flame burners was withdrawn due to reproducibility issues. The use of laser apparatus on the other hand, can be only applied on a very limited area that can impair the applicability of the method [20], [52], [53], although the energy supplied is known. Melt immersion tests produce the most representative temperature changes a refractory can experience in metal forming applications. This is due to the direct contact of the specimen with the melt, but the procedure is very costly [54]. Due to these inefficiencies, experimental results for the cases under consideration are not available. However, the results of the numerical model will be compared with general observations from the literature on hot shocked specimens.

Similarly to the cold shock simulations described in the previous section, the temperature field is approximated numerically using the FEM algorithm. Subsequently the interpolated temperature field is used in the uncoupled peridynamic model to simulate the mechanical response. A structured mesh with a $200 \times 40$ grid was used for the finite element calculations while 52416 particles were used for the spatial discretization of the peridynamic equation of motion. The geometry of the 
model, boundary conditions and application of heat transfer is identical to that described in the previous sections. Since in hot shock the maximum stresses take longer to manifest compared to the cold shock [6], [19], the whole phenomenon is simulated using the static solution.

Both the linear and non-linear heat transfer solutions will be used to simulate thermal induced fracture of a material body that undergoes rapid temperature increase. The heat transfer coefficient is again assumed equal to $50,000 \mathrm{Wm}^{-2} \mathrm{~K}^{-1}$, to simulate the heat exchange between a hot liquid and a cool solid. Such conditions are typical for metal forming and die casting. The temperature variation and a comparison between the temperature field from the linear and the non-linear solution can be found in Figure 9. The temperature fields are extracted from a specimen, initially at ambient temperature $\left(T_{0}=293.15 \mathrm{~K}\right)$, suddenly exposed to an environment of higher temperature $\left(T_{\infty}=873.15 \mathrm{~K}\right)$. It is evident that intense heat transfer takes place at the boundary of the material which is explained from the Biot number, calculated at room temperature as $\mathrm{Bi}=k_{\mathrm{ref}}^{-1} H h=6.74$. In the same figure, the nonlinear solution exhibits steeper temperature gradients, while the linear predicts a smoother transition of the temperature from the boundary to the interior of the material. Furthermore, from the thermomechanical analysis carried out in [19], it can be seen that the nonlinear solution predicts higher compressive stresses at the boundary of the material while the linear solution predicts higher tensile stresses in the interior of the material.
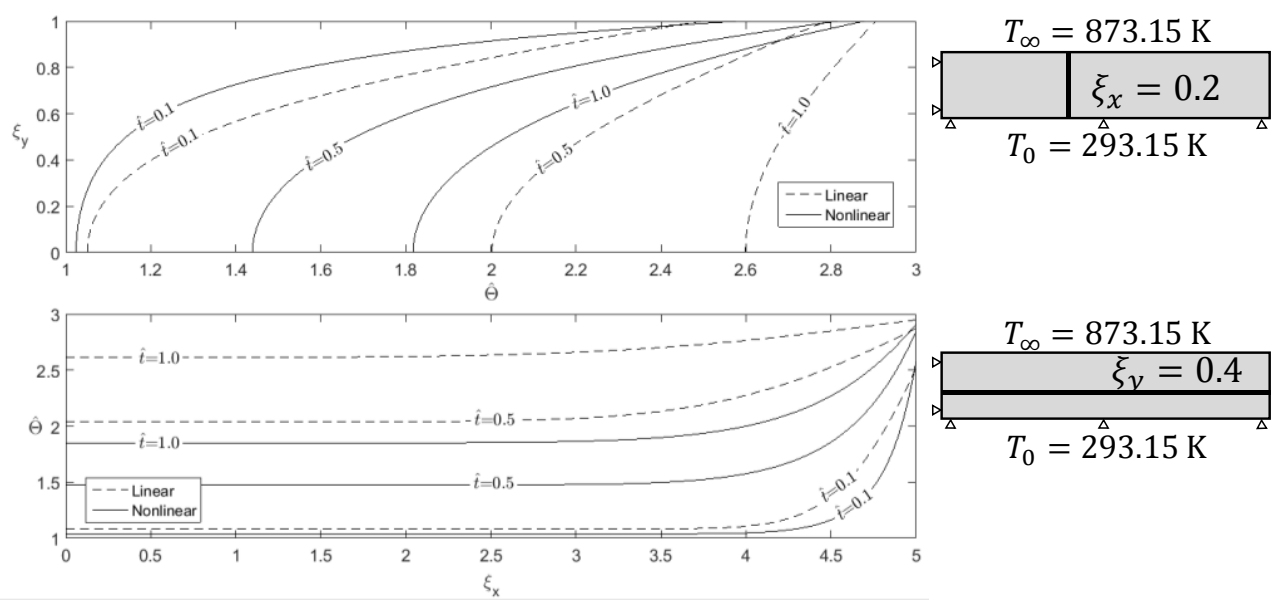

Figure 9: Temperature variation along a vertical and horizontal cross section at various time instants corresponding to initial temperature $T_{0}=293.15 \mathrm{~K}$ and $T_{\infty}=873.15 \mathrm{~K}$.

As a first case, the temperature dependence of the thermal and mechanical material properties is neglected and the properties at room temperature, presented in Figure 2, are implemented for the whole duration of the simulation. To investigate the influence of the surrounding temperature, the simulation was repeated for $T_{\infty}=673.15 \mathrm{~K}, T_{\infty}=773.15 \mathrm{~K}$ and $T_{\infty}=873.15 \mathrm{~K}$.

For all thermal shock magnitudes considered, damage initiated in the interior of the material and propagated towards the upper boundary (Figure 10, left column). Damage is concentrated near the left end, forming a crack at the center of the specimen (left end of one quadrant modelled here). This crack formation is compatible with the statements in the relevant literature [7] and can be 
explained from the tensile stresses developing in the interior of the material. Comparing the crack formed during hot shock with the cracks that emerged during cold shock, it is evident that damage is not restricted to the vicinity of the crack, but peridynamic bonds break in a wider area, leading to material weakening around the crack. Furthermore, only a single crack formed during hot shock that almost completely split the specimen in half.
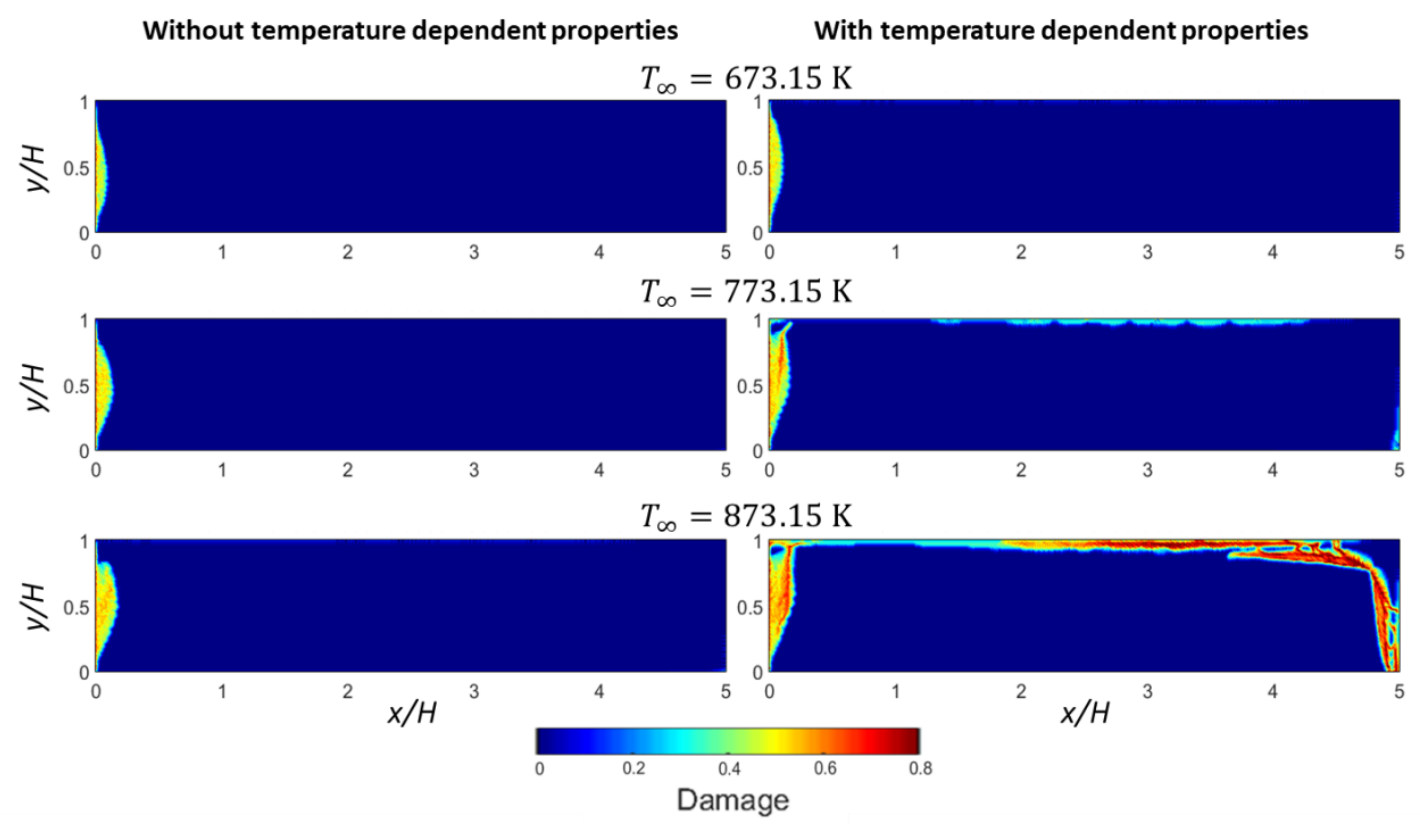

Figure 10: Comparison of damage in a hot shocked specimen after the introduction of the temperature dependent parameters for different shock magnitudes.

As a second case, the temperature dependency of the material parameters from Figure 2, is included. The nonlinear solution of the heat transfer problem, presented in section 4 , is employed for the approximation of the temperature field. Figure 10 illustrates a comparison of the results for the two cases, at the end of the simulation $(\hat{t}=1.00)$. When the temperature dependency is included in the simulations (right column of Figure 10), a total of three damage types can be identified: i) a single crack splits the material in two (similar to the previous case), ii) surface damage appears on the exposed surfaces of the material and iii) a crack at the upper right corner forms when $T_{\infty}=$ $873.15 \mathrm{~K}$. When $T_{\infty}=673.15 \mathrm{~K}$, the introduction of the temperature dependent parameters has led to material weakening in a wider area around the main crack at $x / H=0$, as well as to some limited damage at the heat transfer boundary. Increasing the surrounding temperature to $T_{\infty}=773.15 \mathrm{~K}$ during hot shock, further intensifies the damage at the boundary. At $T_{\infty}=873.15 \mathrm{~K}$, a third type of damage manifests and crack initiates at the corner of the specimen.

A timeline of the damage evolution for a specimen suddenly subjected to an environment at $T_{\infty}=873.15 \mathrm{~K}$, is illustrated in Figure 11. Damage first manifests at the boundary of the material. It initiates at approximately $\hat{t}=0.0107$ and quickly spreads to almost the full length of the boundaries at $\hat{t}=0.0321$. The second damage mechanism that appears is the crack at the boundary 
that initiates at $\hat{t}=0.0519$, followed closely by the last one, the corner crack, that initiates at $\hat{t}=0.0659$. At $\hat{t}=0.1647$ complete fracture of the specimen occurred and the simulation was terminated.

Although both the cold and hot shocked numerical simulations predict damage at the material boundary when the temperature dependence of the material properties is included, the damage mechanisms behind each phenomenon is inherently different. In Figure 12, a close-up of the peridynamic lattice near the top boundary is illustrated for a cold shock and hot shock case. The red lines represent the broken bonds between the particles at the initial stages of the damage. During cold shock, the tensile stresses near the boundary break locally the horizontal bonds, creating the crack nucleation sites. During hot shock on the other hand, it is the vertical bonds that brake, while the horizontal bonds remain intact. This leads to the formation of weakened material layers that at later stages of the simulation, detach from the rest of the solid (see Figure 11 at $\hat{t}=0.1647$ ).
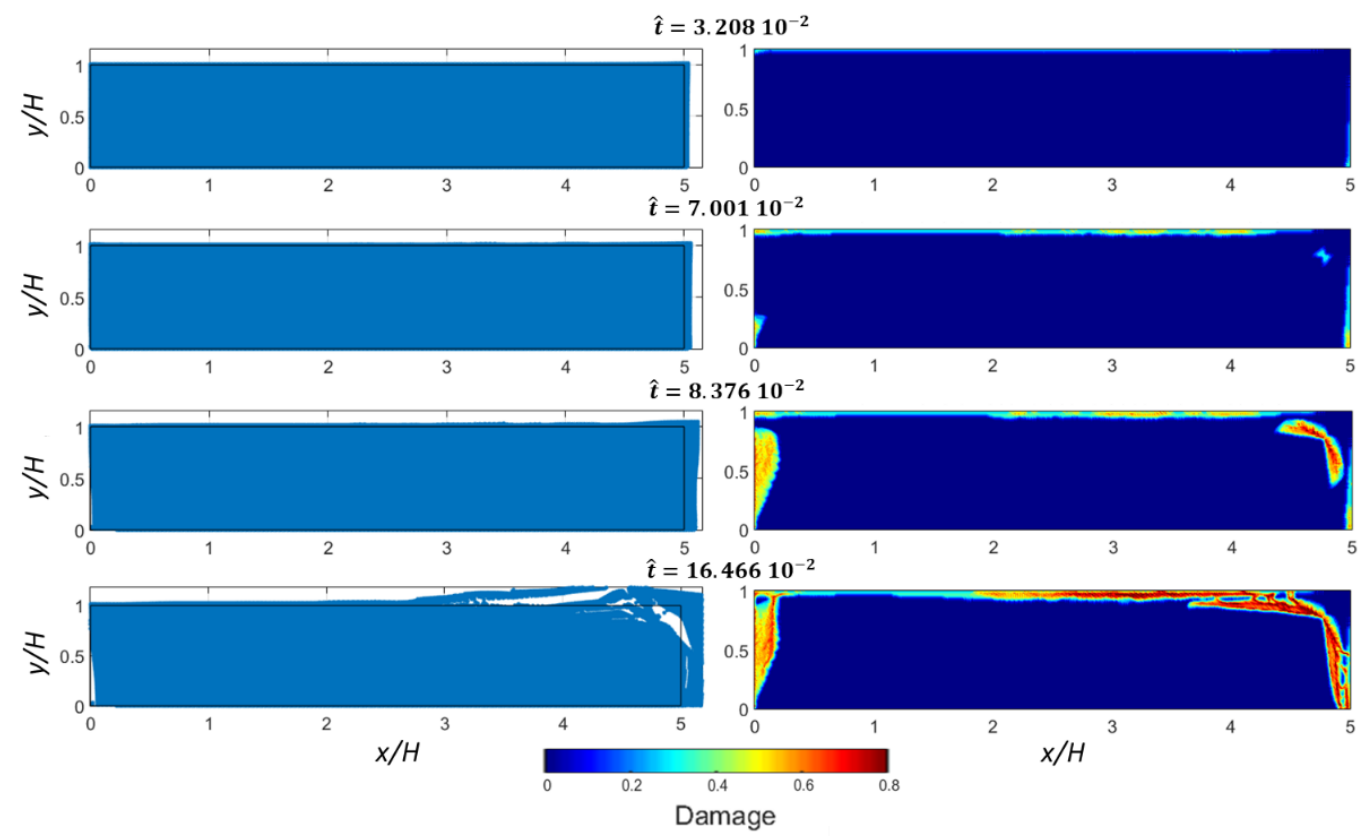

Figure 11: Evolution of damage in a hot shocked alumina specimen from $T_{0}=293.15 \mathrm{~K}$ to $T_{\infty}=873.15 \mathrm{~K}$.

When the temperature dependency of the material parameters is not included in the simulations, surface damage does not manifest at the material surface. This can be attributed to two main reasons. Firstly, at higher temperatures, the material fracture toughness decreases and the material becomes more susceptible to fracture. The second reason becomes apparent considering the thermal stresses that develop in the material during hot shock. When the thermal material properties are not constant, the thermomechanical solution predicts higher compressive stresses near the material boundary compared to when the material properties are not constant [19]. In the literature, it has been pointed out that during hot shock, spalling at the material surface is possible, if the thermal induced compressive stresses are high enough [7], [31]. The fracture mechanism of spalling and the simulated damage at the material boundary appear to have similar characteristics and the present 
methodology could be a good candidate for simulations regarding the material response undergoing a rapid increase in temperature. Referring again to Figure 11, surface damage precedes the formation of a crack near the boundary as well as the corner crack. This agrees with the thermomechanical response of alumina during heating as the compressive stresses near the upper boundary reach their maximum value prior to the tensile stresses in the interior of the material [7], [19].
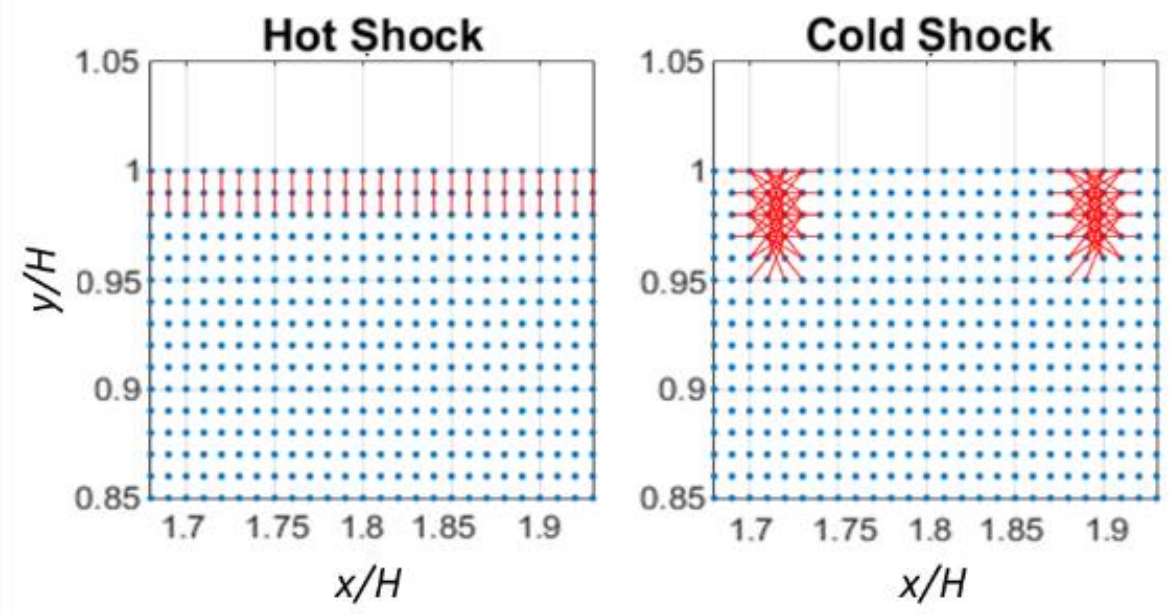

Figure 12: Comparison of broken bonds for a hot and cold shocked specimen.

Interestingly, the corner crack initiates at an angle close to $45^{\circ}$ and subsequently propagates following the boundaries of the specimen. Figure 13 illustrates a close-up of the corner crack along with the temperature field acting on the material. During the thermomechanical investigation of refractories carried out in [19], development of shear stresses near the corners of the domain were observed. Considering a material point at the vicinity of the corner, its temperature will be different compared with neighboring material points in both the vertical and horizontal direction (due to smooth isothermal lines). This temperature difference leads to distortion of the differential areas in close proximity to the corner and the development of shear stresses. This distortion effect is further magnified by the temperature dependence of the thermal expansion coefficient of alumina. The simultaneous combination of shear and compressive stresses on the material points near the corner could explain the fracture during the numerical simulations. 

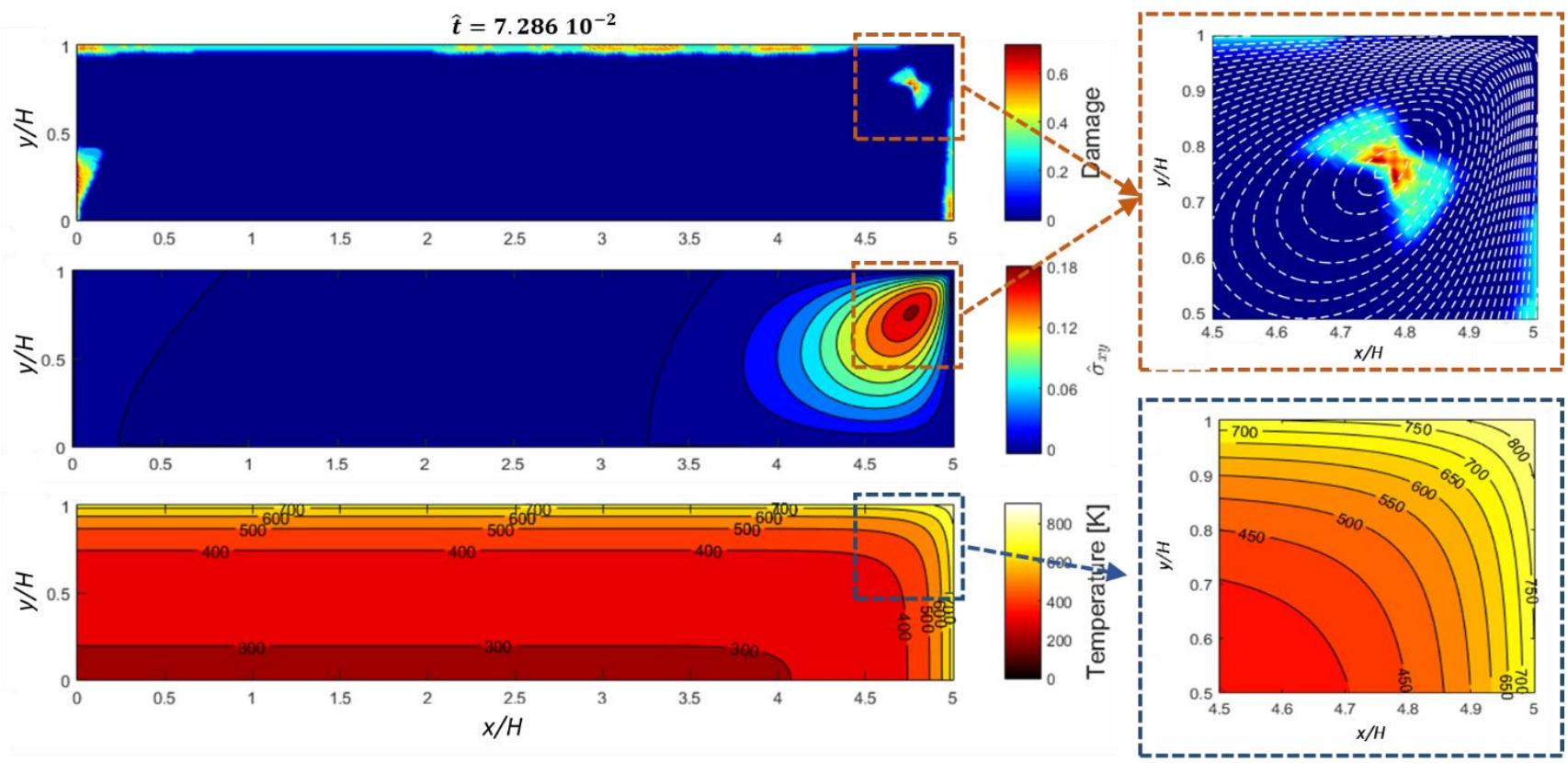

Figure 13: Close-up of the damage (upper plot), the shear stress field (middle plot) and the smooth isothermal contours near the corner of the material (lower plot). $45^{\circ}$

\section{Conclusions}

Numerical simulations of the thermal cracking in ceramics were carried out. A finite element procedure, proposed in [19], was implemented to solve a nonlinear heat transfer model and approximate the temperature field. The temperature field was subsequently used by an uncoupled bond-based peridynamic model to simulate the mechanical response of the material. Both hot and cold shock cases were studied. Thermal and elastic properties corresponding to polycrystalline alumina $\left(\mathrm{Al}_{2} \mathrm{O}_{3}\right)$ and their temperature dependency has been introduced to the simulations.

The peridynamic model was first evaluated with constant material properties using the numerical and experimental results reported in [15] and it successfully simulated the expected crack patterns in cold shocked specimens. After the introduction of the temperature dependent material parameters, the accuracy of the simulations was improved, especially in the prediction of the longest cracks occurring. In hot shock, the model predicted (as expected) cracking of the material originating from its interior, while the temperature dependent parameters allowed for the manifestation of additional damage mechanisms that are in accordance with similar observations in the literature. Finally, the use of the peridynamic model allows for the observation and extraction of dynamic characteristics during crack nucleation.

Acknowledgements: T K Papathanasiou acknowledges support from the European Union FP7 project 'Mechanics of refractory materials at high-temperature for advanced industrial technologies' under contract number PIAPP-GA-2013-609758, for the period 11/2014 - 5/2016. 
Published in Journal of the Journal of the European Ceramic Society, Volume 38, Issue 8, July 2018, Pages 3037-3048

\section{References}

[1] C. P. Jiang et al., 'A study of the mechanism of formation and numerical simulations of crack patterns in ceramics subjected to thermal shock', Acta Mater., vol. 60, no. 11, pp. 4540-4550, 2012.

[2] P. K. Panda, T. S. Kannan, J. Dubois, C. Olagnon, and G. Fantozzi, 'Thermal shock and thermal fatigue study of ceramic materials on a newly developed ascending thermal shock test equipment', Sci. Technol. Adv. Mater., vol. 3, no. 4, pp. 327-334, 2002.

[3] C. B. Carter and M. G. Norton, Ceramic materials: science and engineering. Springer Science \& Business Media, 2007.

[4] X. Wu et al., 'Size effect of thermal shock crack patterns in ceramics and numerical predictions', J. Eur. Ceram. Soc., vol. 35, no. 4, pp. 1263-1271, 2015.

[5] P. Sicsic, J.-J. Marigo, and C. Maurini, 'Initiation of a periodic array of cracks in the thermal shock problem: a gradient damage modeling', J. Mech. Phys. Solids, vol. 63, pp. 256-284, 2014.

[6] J.-C. Han and B.-L. Wang, 'Thermal shock resistance of ceramics with temperature-dependent material properties at elevated temperature', Acta Mater., vol. 59, no. 4, pp. 1373-1382, 2011.

[7] T. J. Lu and N. A. Fleck, 'The thermal shock resistance of solids', Acta Mater., vol. 46, no. 13, pp. 4755-4768, 1998.

[8] H.-A. Bahr, H. Balke, M. Kuna, and H. Liesk, 'Fracture analysis of a single edge cracked strip under thermal shock', Theor. Appl. Fract. Mech., vol. 8, no. 1, pp. 33-39, 1987.

[9] Z. P. Bažant, H. Ohtsubo, and K. Aoh, 'Stability and post-critical growth of a system of cooling or shrinkage cracks', Int. J. Fract., vol. 15, no. 5, pp. 443-456, 1979.

[10] G. A. Schneider and G. Petzow, Thermal shock and thermal fatigue behavior of advanced ceramics, vol. 241. Springer Science \& Business Media, 2013.

[11] I. Jones, 'Impulse response model of thermal striping for hollow cylindrical geometries', Theor. Appl. Fract. Mech., vol. 43, no. 1, pp. 77-88, 2005.

[12] H.-A. Bahr, G. Fischer, and H.-J. Weiss, 'Thermal-shock crack patterns explained by single and multiple crack propagation', J. Mater. Sci., vol. 21, no. 8, pp. 2716-2720, 1986.

[13] G. Carta, I. Jones, M. Brun, N. Movchan, and A. Movchan, 'Crack propagation induced by thermal shocks in structured media', Int. J. Solids Struct., vol. 50, no. 18, pp. 2725-2736, 2013.

[14] J. Li, F. Song, and C. Jiang, 'A non-local approach to crack process modeling in ceramic materials subjected to thermal shock', Eng. Fract. Mech., vol. 133, pp. 85-98, 2015.

[15] J. Li, F. Song, and C. Jiang, 'Direct numerical simulations on crack formation in ceramic materials under thermal shock by using a non-local fracture model', J. Eur. Ceram. Soc., vol. 33, no. 13, pp. 2677-2687, 2013.

[16] B. Bourdin, J.-J. Marigo, C. Maurini, and P. Sicsic, 'Morphogenesis and propagation of complex cracks induced by thermal shocks', Phys. Rev. Lett., vol. 112, no. 1, p. 014301, 2014.

[17] I. Özdemir, W. Brekelmans, and M. G. Geers, 'FE2 computational homogenization for the thermo-mechanical analysis of heterogeneous solids', Comput. Methods Appl. Mech. Eng., vol. 198, no. 3, pp. 602-613, 2008.

[18] M. Nieves, A. Movchan, and I. Jones, 'Asymptotic study of a thermoelastic problem in a semiinfinite body containing a surface-breaking crack and small perforations', Q. J. Mech. Appl. Math., vol. 64, no. 3, pp. 349-369, 2011.

[19] T. K. Papathanasiou, F. Dal Corso, and A. Piccolroaz, 'Thermo-mechanical response FEM simulation of ceramic refractories undergoing severe temperature variations', J. Eur. Ceram. Soc., vol. 36, no. 9, pp. 2329-2340, 2016.

[20] F. Damhof, W. Brekelmans, and M. Geers, 'Experimental analysis of the evolution of thermal shock damage using transit time measurement of ultrasonic waves', J. Eur. Ceram. Soc., vol. 29, no. 8, pp. 1309-1322, 2009. 
[21] A. M. Hofmeister, 'Thermal diffusivity and thermal conductivity of single-crystal $\mathrm{MgO}$ and $\mathrm{Al} 2 \mathrm{O} 3$ and related compounds as a function of temperature', Phys. Chem. Miner., vol. 41, no. 5, pp. 361-371, 2014.

[22] J. G. Hemrick, R. B. Dinwiddie, E. R. Loveland, and A. Prigmore, 'Development of a test technique to determine the thermal diffusivity of large refractory ceramic test specimens', Int. J. Appl. Ceram. Technol., vol. 9, no. 1, pp. 108-114, 2012.

[23] P. Auerkari, Mechanical and physical properties of engineering alumina ceramics. Technical Research Centre of Finland Finland, 1996.

[24] R. Morrell, Handbook of properties of technical and engineering ceramics. Hmso, 1987.

[25] Z. Zhou, F. Song, Y. Shao, S. Meng, C. Jiang, and J. Li, 'Characteristics of the surface heat transfer coefficient for Al 2 O 3 ceramic in water quench', J. Eur. Ceram. Soc., vol. 32, no. 12, pp. 3029-3034, 2012.

[26] W. J. Lee, Y. Kim, and E. Case, 'The effect of quenching media on the heat transfer coefficient of polycrystalline alumina', J. Mater. Sci., vol. 28, no. 8, pp. 2079-2083, 1993.

[27] M. Zaccariotto, F. Luongo, and U. Galvanetto, 'Examples of applications of the peridynamic theory to the solution of static equilibrium problems', Aeronaut. J., vol. 119, no. 1216, pp. 677-700, 2015.

[28] T. L. Anderson, Fracture mechanics: fundamentals and applications. CRC press, 2005.

[29] J. Webb, K. Jakus, and J. Ritter, 'R-curve and subcritical crack growth behavior at elevated temperatures in coarse grain alumina', Acta Mater., vol. 44, no. 6, pp. 2259-2264, 1996.

[30] A. Polyanin, 'Handbook of linear partial differential equations for engineers and scientists. 2002', Bovs Raton ChapmanHallCRC.

[31] W. D. Kingery, 'Factors affecting thermal stress resistance of ceramic materials', J. Am. Ceram. Soc., vol. 38, no. 1, pp. 3-15, 1955.

[32] S. A. Silling, 'Reformulation of elasticity theory for discontinuities and long-range forces', $J$. Mech. Phys. Solids, vol. 48, no. 1, pp. 175-209, 2000.

[33] E. Emmrich and O. Weckner, 'The peridynamic equation of motion in non-local elasticity theory', presented at the III European Conference on Computational Mechanics-Solids, Structures and Coupled Problems in Engineering, 2006.

[34] S. A. Silling, O. Weckner, E. Askari, and F. Bobaru, 'Crack nucleation in a peridynamic solid', Int. J. Fract., vol. 162, no. 1-2, pp. 219-227, 2010.

[35] F. Bobaru, J. T. Foster, P. H. Geubelle, P. H. Geubelle, S. A. Silling, and (first), 'Handbook of Peridynamic Modeling', 2016.

[36] D. De Meo, N. Zhu, and E. Oterkus, 'Peridynamic Modeling of Granular Fracture in Polycrystalline Materials', J. Eng. Mater. Technol., 2016.

[37] E. Madenci and E. Oterkus, Peridynamic theory and its applications, vol. 17. Springer, 2014.

[38] S. A. Silling and E. Askari, 'A meshfree method based on the peridynamic model of solid mechanics', Comput. Struct., vol. 83, no. 17, pp. 1526-1535, 2005.

[39] B. Kilic and E. Madenci, 'Peridynamic theory for thermomechanical analysis', IEEE Trans. Adv. Packag., vol. 33, no. 1, pp. 97-105, 2010.

[40] B. Kilic and E. Madenci, 'Prediction of crack paths in a quenched glass plate by using peridynamic theory', Int. J. Fract., vol. 156, no. 2, pp. 165-177, 2009.

[41] S. Oterkus, E. Madenci, and A. Agwai, 'Fully coupled peridynamic thermomechanics', $J$. Mech. Phys. Solids, vol. 64, pp. 1-23, 2014.

[42] B. Kilic and E. Madenci, 'Coupling of peridynamic theory and the finite element method', $J$. Mech. Mater. Struct., vol. 5, no. 5, pp. 707-733, 2010.

[43] R. W. Macek and S. A. Silling, 'Peridynamics via finite element analysis', Finite Elem. Anal. Des., vol. 43, no. 15, pp. 1169-1178, 2007.

[44] W. Liu and J. W. Hong, 'Discretized peridynamics for linear elastic solids', Comput. Mech., vol. 50, no. 5, pp. 579-590, 2012. 
[45] F. Bobaru, M. Yang, L. F. Alves, S. A. Silling, E. Askari, and J. Xu, 'Convergence, adaptive refinement, and scaling in 1D peridynamics', Int. J. Numer. Methods Eng., vol. 77, no. 6, pp. 852-877, 2009.

[46] M. Nieves, A. Movchan, and I. Jones, 'Analytical model of thermal striping for a microcracked solid', Int. J. Solids Struct., vol. 49, no. 10, pp. 1189-1194, 2012.

[47] E. Oterkus, E. Madenci, O. Weckner, S. Silling, P. Bogert, and A. Tessler, 'Combined finite element and peridynamic analyses for predicting failure in a stiffened composite curved panel with a central slot', Compos. Struct., vol. 94, no. 3, pp. 839-850, 2012.

[48] R. Becker and R. J. Lucas, 'An assessment of peridynamics for pre and post failure deformation', ARMY RESEARCH LAB ABERDEEN PROVING GROUND MD WEAPONS AND MATERIALS RESEARCH DIRECTORATE, 2011.

[49] K. Ravi-Chandar, Dynamic fracture. Elsevier, 2004.

[50] A. J. Rosakis, 'Intersonic shear cracks and fault ruptures', Adv. Phys., vol. 51, no. 4, pp. 11891257, 2002.

[51] W. Zhang, N. Doynov, M. Wolf, O. Dreibati, R. Ossenbrink, and V. Michailov, 'Investigation of the Thermal Shock Behavior of Ceramic Using a Combination of Experimental Testing and FE- Simulation Methods', Adv. Eng. Mater., vol. 15, no. 6, pp. 480-484, 2013.

[52] R. Benz, A. Naoumidis, and H. Nickel, 'Thermal shock testing of ceramics with pulsed laser irradiation', J. Nucl. Mater., vol. 150, no. 2, pp. 128-139, 1987.

[53] S. Akiyama and S. Amada, 'A new method to evaluate the thermal shock resistance of ceramics by laser pulse irradiation', Fusion Sci. Technol., vol. 23, no. 4, pp. 426-434, 1993.

[54] E. Brochen, S. Clasen, E. Dahlem, and C. Dannert, 'Determination of the Thermal Shock Resistance of Refractories'. 